\title{
Parameterized time-frequency analysis to separate multi-radar signals
}

\author{
Wenlong Lu*, Junwei Xie, Heming Wang, and Chuan Sheng \\ Air and Missile Defense College, Air Force Engineering University, Xi' an 710051, China
}

\begin{abstract}
Multi-radar signal separation is a critical process in modern reconnaissance systems. However, the complicated battlefield is typically confronted with increasing electronic equipment and complex radar waveforms. The intercepted signal is difficult to separate with conventional parameters because of severe overlapping in both time and frequency domains. On the contrary, timefrequency analysis maps the $1 \mathrm{D}$ signal into a $2 \mathrm{D}$ time-frequency plane, which provides a better insight into the signal than traditional methods. Particularly, the parameterized time-frequency analysis (PTFA) shows great potential in processing such nonstationary signals. Five procedures for the PTFA are proposed to separate the overlapped multi-radar signal, including initiation, instantaneous frequency estimation with PTFA, signal demodulation, signal separation with adaptive filter and signal recovery. The proposed method is verified with both simulated and real signals, which shows good performance in the application on multi-radar signal separation.
\end{abstract}

Keywords: intercepted multi-radar signal, parameterized timefrequency analysis, demodulation, adaptive filtering.

DOI: $10.21629 /$ JSEE.2017.03.09

\section{Introduction}

Separation of multi-radar signals is a critical process (see Fig. 1) in modern reconnaissance systems (see Fig. 2) that can provide reliable and timely reconnaissance information of location, deployment, disposition and quantity on interested targets [1]. However, the battlefield becomes complicated due to an increasing amount of electronic equipment, leading to severely overlapped multi-radar signals in both time and frequency domains. Moreover, modern radar utilizes complex waveforms developed intentionally [2,3] whose frequency characteristics change over time, to obtain high detection performance and low probabilities of interception and identification. Both reasons make it difficult to detect interleaved radar signals by conventional separation

Manuscript received January 20, 2016.

*Corresponding author. approaches based on the pulse descriptor word [1], i.e., radio frequency, direction of arrival, time of arrival, pulse repetition interval [4] and pulse width.

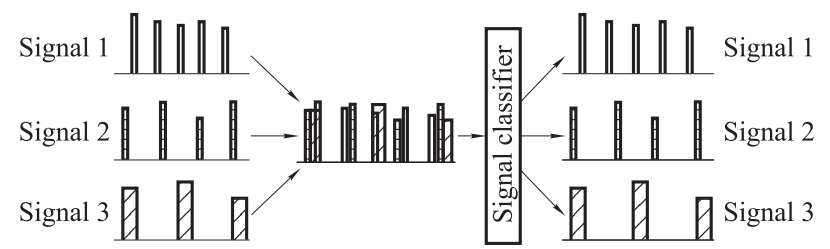

Fig. 1 Flowchart of radar signal separation

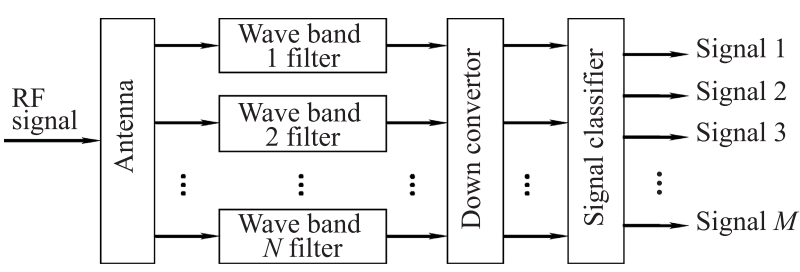

Fig. 2 System structure of typical signal classifier

In despite of detecting the interested signals in the time and frequency domains respectively, reconnaissance receivers should search multi-radar signals in a joint timefrequency domain to distinguish different signals based on their intra-structures. Time-frequency analysis (TFA) offers a new approach in multi-radar signal separation [5]. The intercepted multi-radar signal can be modeled as a multicomponent frequency modulated (FM) signal emitted by different FM sources whose features are characterized with instantaneous frequencies (IF) [6]. Unlike Fourier transform, TFA maps the analyzed signal into a 2D time-frequency plane, i.e., a time-frequency representation (TFR), which provides a better insight into the underlying pattern of the multi-radar signal [7].

TFA is typically performed by two approaches: nonparameterized time-frequency transform (NPTFT) and parameterized time-frequency transform (PTFT), where the difference is whether the transform has signal-dependent 
parameters. A variety of NPTFTs have been presented for the detection and parameter estimation on some similar signals, e.g., short-time Fourier transform (STFT) [8], wavelet transform (WT) [9] and Wigner-Ville distribution (WVD) [10]. Kwok and Jones [11] used an adaptive short-time Fourier transform to estimate IF. Anderson [12] applied the multi-resolution analysis method to the classification of radar pulses in the specific emitter identification problem. Stankovi and Djurovi [13] estimated motion parameters with the spectrogram and WVD. Barbarossa et al. $[14,15]$ studied the cross-term suppression, optimal detection and parameter estimation combined with WignerHough transform (WHT), and further utilized them to analyze the mono- or multi-component linear frequency modulated (LFM) signal. However, these methods often suffer from either poor frequency concentration or interference of cross-terms when dealing with multicomponent FM signals. Furthermore, the absence of prior knowledge typically leads to difficult signal identification and separation in actually complicated battlefields, even worse in existence of the interference.

In contrast with NPTFTs, PTFTs adopt extra parameters related to the FM information of the analyzed signal; this makes the most of emitter prior knowledge and obtains a well-concentrated TFR. The well-concentrated TFR characterizes a good time-frequency pattern of the specific component that is easy to separate from the overlapped multi-radar signal. A number of parameterized time-frequency methods have been proposed in similar applications. As the oldest member, chirplet transform (CT) is widely applied to analyzing the LFM signal with a wellconcentrated TFR [16-18]. A Warblet transform (WBT) [19] is presented to characterize the signal with a periodic IF. Especially, Yang et al. [20] redefined the physical explanation of the parameterized time-frequency methods with frequency rotating and shifting operations. Furthermore, three parameterized kernels, i.e., polynomial kernel [21], spline kernel [22] and Fourier series kernel [23], are proposed to analyze transient vibration signals and achieve good performance.

This paper mainly aims at the application of parameterized time-frequency analysis (PTFA) to multi-radar signal identification and separation. The remainder of the paper is organized as follows. Section 2 reviews the theoretical background concerning signal model and PTFT. Section 3 introduces multi-radar separation with PTFA. In Section 4, the proposed method is verified with experiments on simulated and actual signals. Section 5 draws the conclusion.

\section{Theoretical background}

\subsection{Signal model and IF}

A noisy multi-radar signal [24] can be expressed as

$$
s(t)=\sum_{k=1}^{M} s_{k}(t)+n(t)
$$

where $n(t)$ is white Gaussian noise of power $\sigma^{2}$ and mean of zero. $M$ is the number of the components of the analyzed FM signal. The analytic signal of the $k$ th FM component $s_{k}(t) \in L^{2}(R)$, through the Hilbert transform, is given as follows:

$$
z_{s, k}(t)=s_{k}(t)+\mathrm{j} H\left[s_{k}(t)\right]
$$

where the Hilbert transform of $s_{k}(t)$ is defined as

$$
H\left[s_{k}(t)\right]=\frac{1}{\pi} P . V . \int_{-\infty}^{+\infty} \frac{s_{k}(\tau)}{t-\tau} \mathrm{d} \tau
$$

where P.V. means the integral taken in the sense of Cauchy principal value.

The analytic signal can also be rewritten as

$$
\begin{gathered}
z_{s, k}(t)=\sum_{k=1}^{M} A_{k} \exp \left[\mathrm{j} \phi_{k}(t)\right]+z_{n}(t)= \\
\sum_{k=1}^{M} A_{k}(t) \exp \left[\mathrm{j}\left(\omega_{c, k} t+\int \varphi_{k}(t) \mathrm{d} t\right)\right]+z_{n}(t) .
\end{gathered}
$$

The IF of the signal is $\omega_{k}=\phi_{k}^{\prime}(t)=\omega_{c, k}+\varphi_{k}(t)$, where $\omega_{c, k}$ denotes the carried frequency and $\varphi_{k}(t)$ is the modulation law. $A_{k}(t)$ is the time-varying amplitude of the $k$ th component, which can be treated as a constant compared with its IF.

In this model, when $M=1$, the signal can be termed as a mono-component signal; when $M \geqslant 2$, the signal is referred to as a multicomponent signal. The model defined by (1)-(4) applies to a multi-radar signal with $M$ timevariant components.

\subsection{Generalized parameterized time-frequency transform}

The chirplet transform is an effective method to analyze LFM signals, which is defined as follows:

$$
C T(t, \omega, \alpha, \sigma)=\int_{-\infty}^{+\infty} z(\tau) \psi(\tau, t, \alpha, \sigma) \exp (-\mathrm{j} \omega \tau) \mathrm{d} \tau
$$

where $z(t)$ is the analytic form of $s(t), s(t) \in L_{2}(R)$. $\psi(\tau, t, \alpha, \sigma)$ is a complex window given as

$$
\psi(\tau, t, \alpha, \sigma)=w_{\sigma}(\tau-t) \exp \left[-\mathrm{j} \frac{\alpha}{2}\left(t-t_{0}\right)^{2}\right]
$$

where $t, \alpha \in R$ stand for time and chirping rate, respectively; $w_{\sigma} \in L_{2}(R)$ denotes a window function, which is usually taken as a Gaussian function expressed as

$$
w_{\sigma}(t)=\frac{1}{\sqrt{2 \pi} \sigma} \exp \left[-\frac{1}{2}\left(\frac{t}{\sigma}\right)^{2}\right]
$$


where $\sigma$ determines the length of the Gaussian window.

According to the definition in (5) and (6), the CT can be rewritten [25] as

$$
\begin{gathered}
C T(t, \omega, \alpha, \sigma)= \\
A(t) \int_{-\infty}^{+\infty} \bar{z}(t) w_{\sigma}(\tau-t) \exp (-\mathrm{j} \omega \tau) \mathrm{d} \tau
\end{gathered}
$$

with

$$
\left\{\begin{array}{l}
\bar{z}(\tau)=z(\tau) \Phi^{R}(\tau, \alpha) \Phi^{S}(\tau, t, \alpha) \\
\Phi^{R}(\tau, \alpha)=\exp \left(-\mathrm{j} \alpha \tau^{2} / 2\right) \\
\Phi^{S}(\tau, t, \alpha)=\exp (\mathrm{j} \alpha t \tau) \\
A(t, \alpha)=\exp \left(-\mathrm{j} t^{2} \alpha / 2\right)
\end{array}\right.
$$

where $\Phi^{R}(t, \alpha)$ is the frequency rotation operator and $\Phi^{S}(t, \tau, \alpha)$ is the frequency shift operator. $A(t, \alpha)$ is a complex number with modulus $|A(t, \alpha)|=1$. In the time-frequency analysis, the modulus of the TFR, i.e., $|C T(t, \omega, \alpha, \sigma)|$, is usually of interest and meaning. As indicated in (8) and (9), the CT can be decomposed into three operators: frequency rotation operator, frequency shift operator and STFT operator with the window $w_{\sigma}(t)$, which can be generalized to other transforms.

By the new physical explanation, Yang et al. [26] expanded the definition to the generalized parameterized time-frequency transforms with the similar form as

$$
\begin{gathered}
\operatorname{PTFT}(t, \omega, P)=\int_{-\infty}^{+\infty} \bar{z}(t) w_{\sigma}(\tau-t) \exp (-\mathrm{j} \omega \tau) \mathrm{d} \tau \\
\left\{\begin{array}{l}
\bar{z}(\tau)=z(\tau) \Phi_{P}^{R}(\tau) \Phi_{t, P}^{S}(\tau) \\
\Phi_{P}^{R}(\tau)=\exp \left[-\mathrm{j} \int \varphi_{P}(\tau) \mathrm{d} \tau\right] \\
\Phi_{t, P}^{S}(\tau)=\exp \left[\mathrm{j} \tau \phi_{P}(t)\right]
\end{array}\right.
\end{gathered}
$$

where $w_{\sigma} \in L_{2}(R)$ denotes non-negative, symmetric and normalized real window and $\sigma$ controls the window length; $\Phi_{P}^{R}(\tau)$ and $\Phi_{t, P}^{S}(\tau)$ are rotation operator and shift operator, respectively.

\section{Signal separation with PTFA}

The proposed method can be mainly divided into five steps: (i) initiation, (ii) instantaneous frequency estimation with PTFA, (iii) signal demodulation, (iv) adaptive filtering and (v) recovering signal. Fig. 3 illustrates the procedure of signal separation with PTFA.

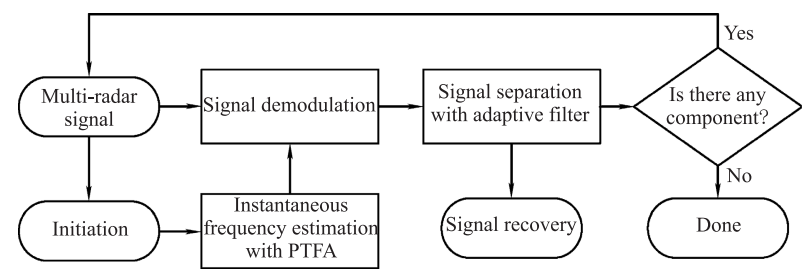

Fig. 3 Flowchat of multicomponent signal separation (i) Initiation

When approximating the ridge of the TFR with a proper kernel function, it is easy to obtain the desired transform parameters of PTFA. However, ridge extraction strongly depends on the clarity of TFR, which is always a main problem of TFA in applications. To obtain more prior knowledge for kernel selection, we usually adopt the results of NPTFT for initiation. Among the NPTFAs, STFT is the simplest and most commonly used method. Compared with other TFA methods, STFT has two advantages, one of which is that little computation is added in radar signal separation. The other is that it has a wide-range adaption, unlike the quadratic TFA-based method that is always accompanied with the problems of cross-terms in multicomponent signals and sensitivity to noise. Nevertheless, the constant resolution of the STFT is typically not sufficiently high to characterize the signal properly when the analysis window does not match. To overcome this deficiency, we utilize a series of windows with different window widths at each time, i.e., an ASTFT [11]. If the window at each time instant is matched to the signal characteristics of that instant, a vivid TFR can be shown by the transform.

The ASTFT is identical to the STFT, except that the analysis window is adaptive to the signal. Different windows are employed at different times to achieve a good TFR over the whole signal. $\left\{w_{1}, w_{2}, \ldots, w_{N}\right\}$ are a set of windows with $\left\|w_{i}\right\|^{2}=1$, for $i=1,2, \ldots, N$. The ASTFT of the signal $z(t)$ can be given as

$$
\begin{gathered}
\operatorname{ASTFT}(t, \omega)= \\
\int_{-\infty}^{+\infty} z(t) w_{i}(\tau-t) \exp (-\mathrm{j} \omega \tau) \mathrm{d} \tau .
\end{gathered}
$$

An adaption rule is needed to determine the optimal window from $N$ candidates. The most popular adaption criteria are based on maximum correlation and minimum entropy, where the latter requires more computation effort than the former. Therefore, a maximum correlation criterion [22] is adopted here, with

$$
\begin{gathered}
i_{C M}(t, f)= \\
\arg \max _{1 \leqslant i \leqslant N}\left|\int z(\tau) w_{i}(\tau-t) \exp (-\mathrm{j} \omega \tau) \mathrm{d} \tau\right| .
\end{gathered}
$$

The maximum correlation rule indicates that the optimal window is the one that maximizes the projection of the signal onto the window. The window set depends highly on the nature of the signals to be analyzed. The computation of ASTFT will be $N$ times as the amount of their corresponding STFT. No set will perform well in all applications. Other possible sets include windows with different chirp rates and windows matched to different classes 
of signals. However, a greater number of parameters involve more computation effort as well as greater accuracy; a trade-off must be made.

(ii) Instantaneous frequency estimation with PTFA

The transform will approach to the maximum energy ridge when the intercepted signals match the parameterized kernel constructed with prior knowledge of the specific radar. However, the parameterized method is practically confronted with the situations where not the entire prior knowledge of the specific radar is known. More reasonably, the intelligence about the specific radar is partly obtained in many ways, including reconnaissance, wiretap and espial. The IF estimation of multi-radar signals based on the parameterized method may vary due to the known prior information. Therefore, the IF estimation of multiradar signals is discussed in three cases.

Case 1 All detailed information is known.

This is an ideal case, where all information about the specific radar emitter, e.g., radar signal, is obtained in advance. The parameterized kernel is constructed with prior knowledge. The parameterized transform of the intercepted signal is performed with the suitable kernel to find the energy ridge of the intercepted signal in the timefrequency plane. The polynomial function is taken as an example for IF approximation.

$$
z(t)=A(t) \exp \left[\mathrm{j}\left(2 \pi f_{c} t+2 \pi \sum_{k=2}^{n+1} \frac{a_{k-1}}{k} t^{k}\right)\right]
$$

where $\left\{a_{1}, a_{2}, \ldots, a_{n}\right\}$ are parameters of the polynomial phase. According to Weierstrass approximation theorem, it is reasonable to adopt the polynomial phase signal to characterize a large number of FM signals. The IF of the modeled signal can be expressed as

$$
I F(t)=f_{c} t+\sum_{k=2}^{n+1} a_{k-1} t^{k-1} .
$$

For the signal in (14), the polynomial chirplet transform (PCT) is proposed to generate the well-concentrated TFR. To identify the IF of the specific radar signal from the multi-radar signal, the integral of the modules of PCT is performed along the IF function, i.e., $I F(t)$, which is defined as

$$
W=\int_{I F}\left|P C T\left(t, \omega ; \alpha_{1}, \alpha_{2}, \ldots, \alpha_{n}\right)\right| \mathrm{d} l>\delta
$$

where $\delta$ is a predefined threshold. When $W$ is greater than $\delta$, we assume the existence of the specific radar signal; otherwise, we assume no specific radar signals. In this case, the computation is similar as their corresponding STFT with the same widow length.
Case 2 Partial prior knowledge is known.

Compared with Case 1, this case is more common in real applications. The detailed information of the specific radar signal is unknown or difficult to determine in advance. By the intelligence and technology analysis, we can only obtain partial prior knowledge about the signal, e.g., the pattern of the signal. Moreover, we may also know that the signal passes through a few frequency points, e.g., $\left(t_{0}, f_{0}\right)$, $\left(t_{1}, f_{1}\right), \ldots,\left(t_{n}, f_{n}\right)$. The selected model based on prior knowledge is able to approximate the characteristics of the IF, whereas the parameters are unknown.

In this situation, signal identification has to be performed along with parameter estimation. The parameters used to be obtained by iteratively fitting the extracted energy ridge from the TFR. However, this method typically suffers from the improper initial TFR. Even though the iterative fitting has the capability of reducing the random errors, the poor concentration of the initial TFR or the significant interference of the noise will destroy the estimation. Therefore, it is important to achieve the proper parameters for the PCT without considering the TFR, especially at a low signal-to-noise ratio (SNR). A spectrum concentration index (SCI) method [7] is proposed to avoid such a problem.

The polynomial kernel applied in SCI can be expressed as

$$
\kappa\left(t, \alpha_{1}, \alpha_{2}, \ldots, \alpha_{n}\right)=\sum_{k=2}^{n+1} \alpha_{k-1} t^{(k-1)} .
$$

According to the SCI, parameter estimation can be translated into a single-objective optimization problem with the constrained conditions of $\left(t_{0}, f_{0}\right),\left(t_{1}, f_{1}\right), \ldots$, $\left(t_{n}, f_{n}\right)$, which is given as

$$
\begin{gathered}
\left\{\tilde{\alpha}_{1}, \tilde{\alpha}_{2}, \ldots, \tilde{\alpha}_{n}\right\}=\arg \max _{\omega} \operatorname{SCI}\left(\alpha_{1}, \alpha_{2}, \ldots, \alpha_{n}\right), \\
\text { s.t. }\left(t_{0}, f_{0}\right),\left(t_{1}, f_{1}\right), \ldots,\left(t_{n}, f_{n}\right) .
\end{gathered}
$$

It is worth noting that all situations with partial prior knowledge can be solved with the optimal model. When the time-frequency pattern of the specific radar signal is unknown, the problem is to search the best form that can fit the signal with the constrained frequency points. When the time-frequency points are unknown, the optimization problem will search its optimal solution without restriction.

The nonlinear optimization problem in (18) can be solved by various techniques, e.g., genetic algorithm, neural network, particle swarm optimization (PSO) and bee colony algorithm. The estimated parameters based on the SCI optimization can be used to characterize the IF of multi-radar signal as well as in Case 1. In this case, the 
computation differs largely in different applications. However, when calculating such an optimal model, we usually set a largest iteration time, e.g., $R$, in case that iteration can not converge. In every iteration, the computation is just similar as its corresponding Fourier transform. Therefore, the total computation is $R$ times as Fourier transform at most.

Case 3 No prior knowledge is known.

In this case, no prior knowledge can be utilized for the construction of parameterized kernel. Multi-radar signal identification can be performed in two ways: one is to degrade the parameterized method to be a nonparameterized one, and the other is to take the results of nonparameterized methods as the prior knowledge for PTFA. The results in initiation can provide initial energy ridge along the IF and prior knowledge for kernel selection.

For a multi-radar signal, PCT with the SCI optimization algorithm is still a suitable method to obtain a wellconcentrated TFR for each component. We can also obtain the matched transform parameters by maximizing the SCI. Hence, the transform parameters estimation turns to be an optimization problem without restriction as follows:

$$
\left\{\tilde{P}_{1}, \ldots, \tilde{P}_{k}\right\}=\arg \max _{\omega} \operatorname{SCI}_{(k)}(P)
$$

where $k$ stands for the number of the components, $\tilde{P}$ stands for the estimated transform parameters. According to (19), for a monocomponent signal, the optimization is a single-objective optimization problem, while it is a multiobjective optimization problem for the multicomponent signal. In this case, the computation is similar as that in Case 3.

The search region can be determined with the following two aspects: i) observation based on the NPTFT; ii) trial and error. In practice, it is suggested to begin with a larger search region at first, and then reduce the region gradually. But such procedure should be balanced with the desired convergence rate. Besides, the parameterized timefrequency analysis is the most time-consuming procedure in the whole algorithm. The computation burden will be of much difference due to their prior knowledge and searching strategy.

(iii) Demodulation

Before filtering the signal, demodulating the observed signal in terms of the interested component is an important job. It can be learned in (10) and (11) that the rotation operator implements signal energy redistribution along the difference between kernel function and real IF in the timefrequency plane, and the shift operator is to move the redistributed energy to the coordinates indicated by the kernel function. When the kernel approaches the time-variant term of the real IF, the occupied bandwidth will decrease; otherwise, the occupied bandwidth will increase when the kernel becomes far away. Ideally, the targeted component is rotated from a nonstationary signal to be a stationary one, where its IF trajectory will be a straight line parallel to the time axis in the time-frequency plane. Seen from the frequency axis, the energy of the interested component is concentrated at a frequency point. In this case, the PTFA attains the most concentrated TFR after the shifting process. On the contrary, the noise content is still evenly distributed across the whole spectral band. The non-interested components are modulated in a broader bandwidth.

(iv) Adaptive filter

Filtering the demodulated signal is the last step before signal recovery. After demodulation, the interested conponent can be treated as stationary, and the design of the filter will become very easy. For a filter, there are two key parameters: central frequency $\omega 0$ and bandwidth $B$. The central frequency of the filter can be determined based on the frequency of the demodulated signal with the peak amplitude [24], i.e.,

$$
\omega_{i}=\arg \max _{\omega}\left[G_{s i}\left(\omega ; \tilde{P}_{i}\right)\right]
$$

The bandwidth $B$, however, is usually a problem. Many papers used to choose a constant bandwidth for the filter based on experience. There are two deficiencies in this method. On one hand, the estimated IF can not be always the same as the real IF of the signal anywhere, i.e., there is still a little 'distance' from the actual IF of the signal to the estimated IF when limited orders are utilized to approximate the IF of the signal. Therefore, a narrow bandwidth can not always cover the whole energy of the subsignal and this will result in signal distortion. On the other hand, a broader bandwidth usually covers more non-interested components, including noise, which will become serious in crossed IF cases and strong noise cases.

The notion of variable bandwidth can be used to solve this problem [27]. On the assumption that the amplitude of the complex signal is constant, the amplitude of the demodulated signal can be treated as approximating extent between estimated IF and true IF. The ridge concentrates at nearly one point seen from frequency axis, whereas maximum values are different along the time axis. There are two reasons for the different ridge values along the time axis. One is the fact that the estimated parameters cannot guarantee the parameterized time-frequency analysis to accurately approximate the real IF of the component of interest. As is known, the estimated IF still has a short 'distance' from the real IF regardless of the optimality of the parameters. Thus, the energy of the demodulated signal projected on the constant frequency is different along the time axis due to the distance between the real IF and estimated IF. 
The other reason for the different ridge values along the time axis is the existence of crossed points in the multicomponent signal. The energy at a crossed point is a sum of different components, which is naturally larger than that elsewhere, particularly when components are slightly different. So when designing the filter, the bandwidth can be adaptive with the amplitudes along the time axis. There are many ways to determine the relations between bandwidth and amplitude $[28,29]$, which varies with different applications. Here, we simply take a filter with a Gaussian window as an example:

$$
w_{\sigma}(\omega)=\frac{1}{\sqrt{2 \pi} \sigma} \exp \left[-{\frac{\left(\omega-\omega_{0}\right)^{2}}{2 \sigma^{2}}}^{2}\right]
$$

where $\omega_{0}$ and $\sigma$ determine the filter's central frequency and bandwidth, respectively. When the time-frequency parameters of the component of interest have been obtained by SCI optimization, the PCT/WBT/GWT can be applied to analyze the signal; theoretically, the energy ridge will appear along the optimal path determined by these parameters in the time-frequency plane. However, the ridge values along the time axis differ from each other due to the error between the approximated and real IF, as well as the existence of crossed components. Moreover, the difference between ridge values can be large at some time points. The bandwidth of the filter should be narrow at high-ridge places because of the accurate approximation or crossed points. In contrast, a wide bandwidth should be utilized at low ridges to cover a greater portion energy of the component of interest. The bandwidth can be determined with an inverse proportion, logarithmic inverse proportion, or exponential inverse proportion; the results are a trade-off between IF accuracy and the amount of energy of the component of interest covered. The digital finite impulse response (FIR) filter can be applied to the demodulated signal components, where the order of the filter is determined by the pass-band and stop-band. The bandwidth of the filter is updated with real-time parameters; the filter can be considered open-loop adaptive. If we allow the maximum pass-band to be $10 \%$ of the half sampling frequency $\omega_{0}$, i.e., $\sigma_{0}$, it will appear at the minimum ridge $A_{\min }$ along the time axis. At other time points, the bandwidth is inversely proportional to the amplitude $A$, i.e.,

$$
\sigma=\frac{A_{\min }}{A} \sigma_{0} .
$$

The obtained component after filtering is

$$
z_{s i}(t)=A_{s i} \exp \left[\mathrm{j}\left(\omega_{i} t+\delta(t)\right)\right]+e(t)
$$

where $\delta(t)$ is significantly small as the parameters are properly estimated by optimizing SCI. $e(t)$ includes the noise and a small portion of unwanted components. The latter might be included when the initial frequencies of components are close to each other or the IF trajectories of components are intersected after demodulation.

(v) Recovering

The filtered component is recovered with the estimated parameters [24] as

$$
\tilde{z}_{s i}(t)=z_{s i}(t) \exp \left[\mathrm{j} \int \phi_{\tilde{P}_{i}}(t) \mathrm{d} t\right]
$$

and then the signal residual is computed as

$$
z(t)=z(t)-\tilde{z}_{s i}(t) .
$$

The above procedure is performed iteratively until no interested signal exists. The algorithm starts with the radar signal of large energy in the process of signal identification and separation. The iterative process will stop when the overall energy of the residual becomes quite small, which indicates no signal left.

\section{Applications on intercepted radar signals separation}

First, in order to compare the performance of different traditional transforms at different SNRs, a polynomial phased signal with constant amplitude is given as

$$
\begin{aligned}
x(t)= & \sin \left(1.254 t+1.158 \times 10^{-3} t^{2}-\right. \\
& \left.3.517 \times 10^{-7} t^{3}\right)+n(t)
\end{aligned}
$$

whose IF is $f(t)=0.1996+0.3686 t+1.6792 t^{2}$. The sampling frequency is normalized and the sampled points are 2000 . In order to compare the noise tolerance of the proposed method with other methods, the signal is masked with white Gaussian noise whose SNRs are $0 \mathrm{~dB}$ and $-10 \mathrm{~dB}$, respectively. Fig. 4 provides the Fourier transform (FT) spectrums of the analyzed signal at two SNRs. It can be learned that the noise is too heavy to distinguish from the target component when $\mathrm{SNR}=-10 \mathrm{~dB}$.

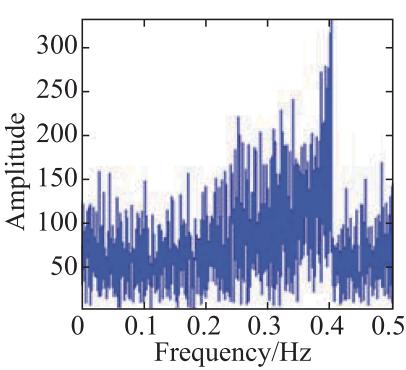

(a) $\mathrm{SNR}=0 \mathrm{~dB}$

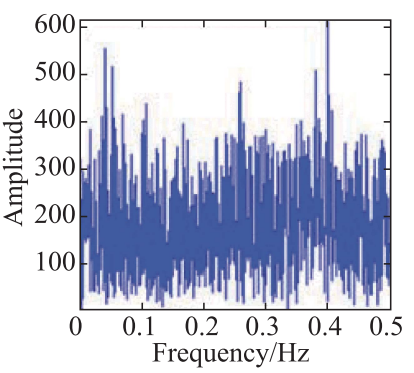

(b) $\mathrm{SNR}=-10 \mathrm{~dB}$
Fig. 4 FT spectrums of the signal at two SNRs

The TFRs generated by STFT, WVD and PCT are shown in Figs. 5-7. Thereinto, the STFT shows poor time-frequency concentration and fails in respresenting the 
time-varying IF of the non-stationary signal due to its constant time-frequency resolution. The TFR of STFT at $0 \mathrm{~dB}$ only shows an IF silhouette while the IF signature of the signal is totally covered by noise at $-10 \mathrm{~dB}$ in Fig. 5 . The WVD can achieve the best time-frequency concentration for the single linear frequency modulated signal, yet it is unable to suppress crossed terms. As shown in Fig. 6, self-crossed terms act as main interference at $0 \mathrm{~dB}$ and mutual-crossed terms between the NLFM signal and noise dominate the smeared TFR at the lower SNR. In the PCT, accurate approximation of the IF largely depends on a clear energy ridge in the time-frequency plane, which is not suitable for the low-SNR signal. Therefore, the clear IF at $0 \mathrm{~dB}$ becomes smeared in the TFR at $-10 \mathrm{~dB}$, as shown in Fig. 7.

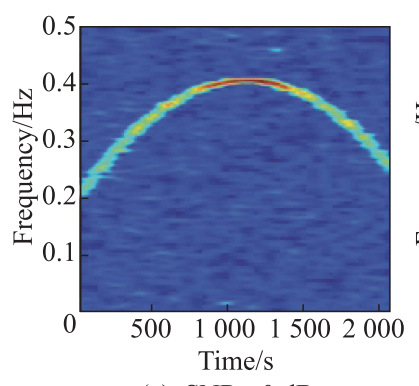

(a) $\mathrm{SNR}=0 \mathrm{~dB}$

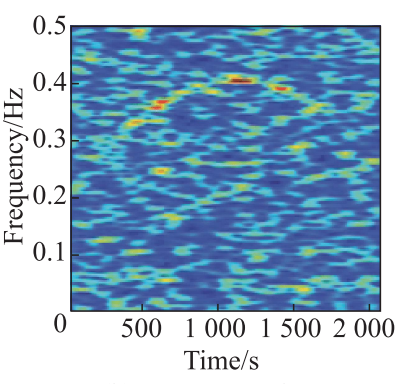

(b) $\mathrm{SNR}=-10 \mathrm{~dB}$
Fig. 5 STFT at different SNRs

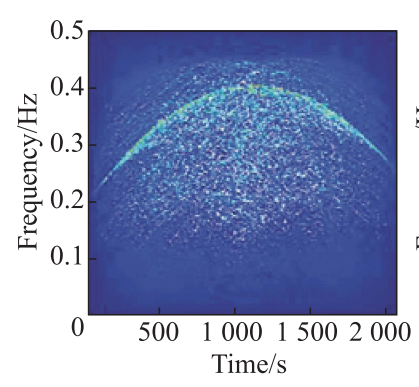

(a) $\mathrm{SNR}=0 \mathrm{~dB}$

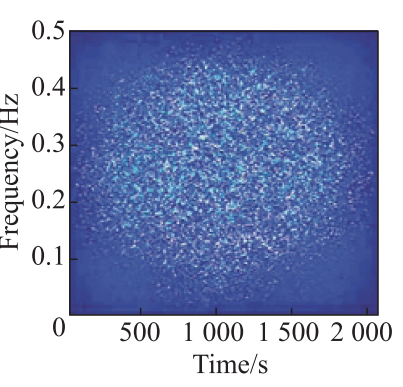

(b) $\mathrm{SNR}=-10 \mathrm{~dB}$
Fig. 6 WVD at different SNRs

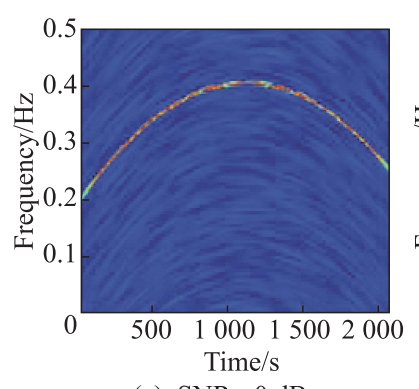

(a) $\mathrm{SNR}=0 \mathrm{~dB}$

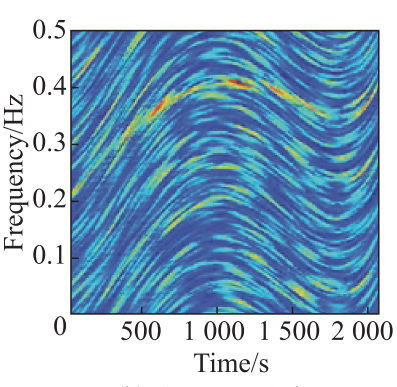

(b) $\mathrm{SNR}=-10 \mathrm{~dB}$
Fig. 7 PCT at different SNRs

In contrast with conventional transforms, the SCI method is adopted. The population size of PSO is set to 30 , and the search regions are set to $[-0.1,0.1]$ and
[-0.01, 0.01] for $\alpha_{1}, \alpha_{2}$ according to sampling frequency. Estimated parameters at both SNRs are obtained by an iterative search and listed in Table 1.

Table 1 Estimated parameters at different SNRs

\begin{tabular}{cccc}
\hline Parameter & SNR/dB & $\tilde{a}_{1}$ & $\tilde{a}_{2}$ \\
\hline \multirow{2}{*}{ Value } & 0 & $1.1571 \mathrm{e}-3$ & $-3.5155 \mathrm{e}-7$ \\
& -10 & $1.1610 \mathrm{e}-3$ & $-3.5280 \mathrm{e}-7$ \\
\hline
\end{tabular}

With estimated parameters, the component energy is largely concentrated at both SNRs. Then the target component is extracted by a band-pass filter with $1 \%$ bandwidth of sampling frequency. Fig. 8 describes the TFRs of the extracted component at different SNRs. Both of them accurately characterize the IF of the analyzed signal.

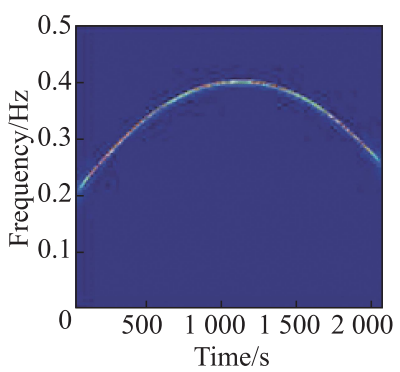

(a) $\mathrm{SNR}=0 \mathrm{~dB}$

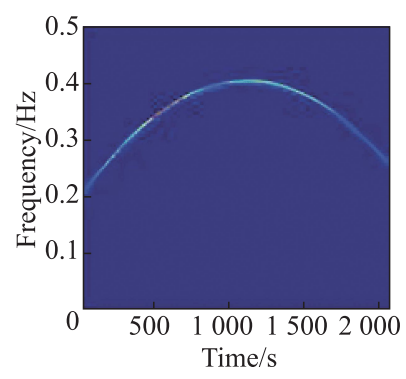

(b) $\mathrm{SNR}=-10 \mathrm{~dB}$
Fig. 8 TFRs of the extracted component at different SNRs

Second, we consider a two-component synthetical signal as (27), i.e. $s_{1}(t)$ and $s_{2}(t)$, where $n(t)$ is white Gaussian noise. In our example the sampling frequency is set to be $100 \mathrm{~Hz} . s_{1}(t)$ is $20 \mathrm{~dB}$ larger than $s_{2}(t)$ and $n(t)$. Besides, both of the components have an additional part of $\sin (2 t)$ indicating oscillation of the signal due to the instability of the signal generator. The ASTFT is employed to provide prior knowledge about the number of components.

$$
\begin{gathered}
z(t)=s_{1}(t)+s_{2}(t)+n(t)= \\
4 \sin \left\{2 \pi\left[2+5 t-0.15 t^{2}+0.2 t^{3}+0.05 \sin (20 t)\right]\right\}+ \\
2 \sin \left\{2 \pi\left[5+35 t+3 t^{2}-t^{3} / 15+0.05 \sin (20 t)\right]\right\}+n(t) \\
0 \leqslant t \leqslant 10 \mathrm{~s} .
\end{gathered}
$$

Fig. 9(a) illustrates synthetical signal of $s_{1}(t)$ and $s_{2}(t)$ mixed with noise. Besides, Fig. 9(b) shows the instantaneous frequencies of the two components calculated from their formulas. All TFRs are obtained by PCT, which uses the estimated parameters to obtain an improved TFR. The phase order is set to 3. A zero-phase digital filter is used to keep the phase of the filtered component unchanged, whose pass-bands are adaptive around estimated central frequency. The attenuate rates of the pass-band and the stop-band are set to be $3 \mathrm{~dB}$ and $60 \mathrm{~dB}$, respectively, which are determined by specific need of different applications. 


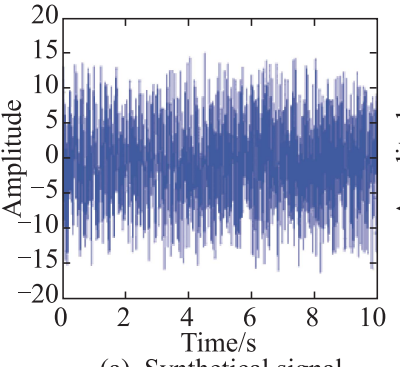

(a) Synthetical signal

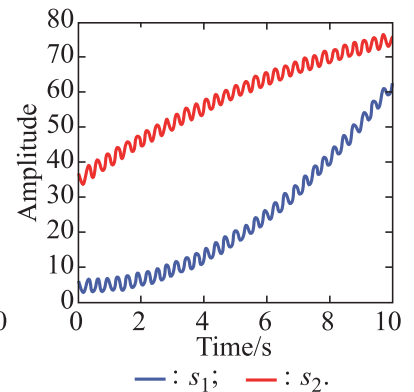

(b) Instantaneous frequencies
Fig. 9 Synthetical signal and their instantaneous frequencies

Fig. 10 shows the results of the two extracted components' TFRs of the proposed component extraction procedure. The two recovered signals have a litte difference due to their SNRs. It can be seen that the proposed algorithm could extract the respect component accurately. Compared with the IFs of $s_{1}(t)$ and $s_{2}(t)$ in Fig. 9(b), the estimation ignores the details on the IF law due to limited phase order, which will become more serious when the oscillation is large. Obviously, a narrow constant band-pass filter will lead to signal distortion, whereas a wide constant one will introduce more noise. As a counterpart, an adaptive filter can reserve as much signal as possible with less noise.

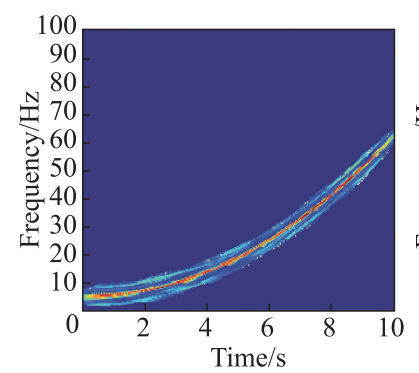

(a) TFR of 1 st component

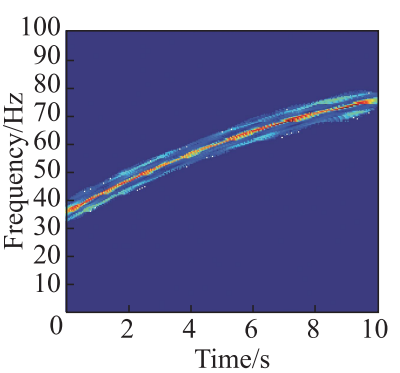

(b) TFR of 2 nd component
Fig. 10 TFRs of the filtered signal component

To illustrate the effectiveness of the proposed method on real signals with more than two components, we use an example of intercepted radar signals (Fig. 11(a)) obtained in the microwave laboratory. In the experiment, four radars emit their pulse signals which have the same pulse repetition frequencies, similar radio frequencies and different radiation powers and pulse widths. Moreover, a jammer radiates white noise around the carrier frequencies of four radar signals. This setup is to simulate the overlapped multi-radar signals with noise in real battlefield. The sampling frequency of the intercepted signal after down-conversion is $50 \mathrm{MHz}$ and the sampling time is $50 \mu$ s. The size of the analysis window is set to 1024 . The received signal includes several signals from diffe- rent radars as well as noise contamination with an SNR of $10 \mathrm{~dB}$. The intercepted signal is a multicomponent signal comprised of not only LFM components but also NLFM components. It can be seen that components are interleaved in both time and frequency domains, which is hardly separated with the conventional method (Fig. 11(b)). The ASTFT can help find four subsignals, whereas components have poor concentration due to the severely smeared TFR. The ASTFT adjusts the window length to achieve the adaptive resolution. The result of STFT provides prior known for kernel selection and initial energy ridge for iterative approximation. However, the concentration of the TFR is still not satisfying.

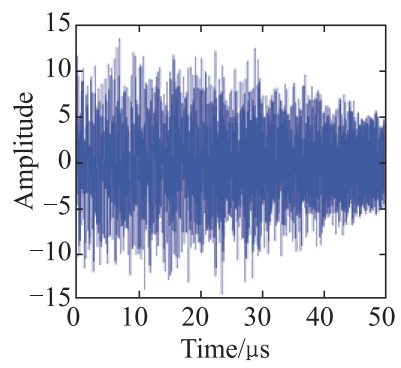

(a) Signal in time domain

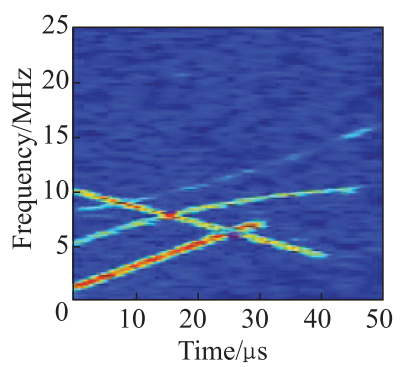

(b) ASTFT of radar signal
Fig. 11 Intercepted radar signal

In the case where the components are of sufficient separation with others, a minimum strip region enclosing the component of interest is selected based on the prior knowledge of the TFD of ASTFT. On the other hand, when the IF laws of the components are intersected, the ridge of the wanted component needs to be adjusted to avoid the interference of unwanted components. The ridge is first extracted from the strip area, and the interval of the ridge around the intersection point of two IFs is removed. Then, the missing values of the ridge in the interval are calculated by using a linear interpolation. According to the iteration, the ridge can be refined to close the true IF of the interesting component. Fig. 12 provides TFRs of the extracted subsignals, which shows that the desired components are extracted successfully. The four separated radar signals are indicated in Fig. 12(a)-Fig. 12(d), respectively, and their estimated coefficients are shown in Table 2. Besides, the estimated IFs are compared with their real IFs and the errors are also listed in the table. The error is defined as the mean square error (MSE) [30] between the estimated IF and real IF, and given as follows:

$$
\mathrm{MSE}=\frac{\left\|\tilde{f}_{\text {inst }}(t)-f_{\text {inst }}(t)\right\|_{2}}{\left\|f_{\text {inst }}(t)\right\|_{2}}
$$

where $\tilde{f}_{\text {inst }}(t)$ and $f_{\text {inst }}(t)$ indicate estimated IF and real IF, respectively. It can be seen from the figure that more 
energy is reserved around lower ridge values, which will provide better fidelity for the recovered signal. The errors of the estimated IFs become larger along with their decreasing energy, which verifies the necessity of an adaptive filter for as much as component energy.

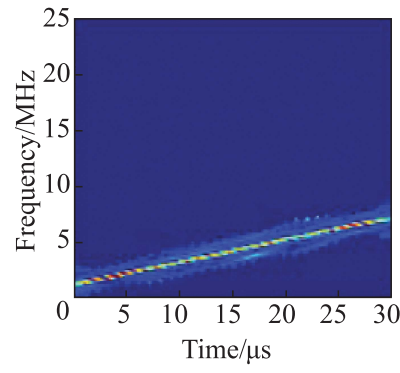

(a) TFR of Signal 1

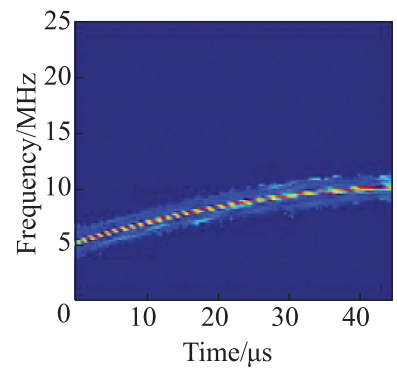

(c) TFR of Signal 3

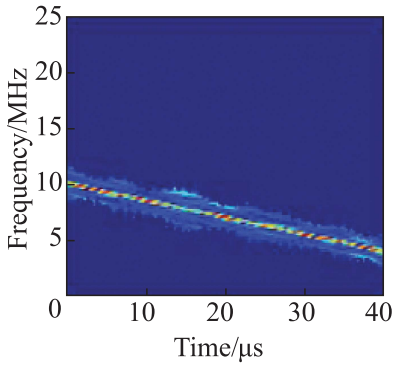

(b) TFR of Signal 2

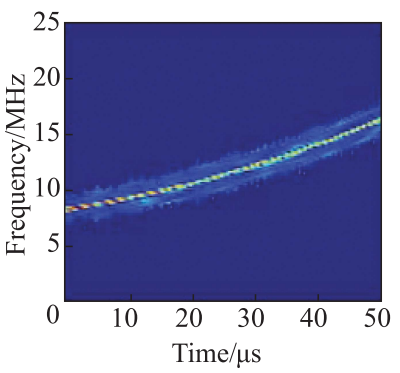

(d) TFR of Signal 4
Fig. 12 TFRs of the separated radar signals

Table 2 Estimated coefficients of the IFs

\begin{tabular}{cccccc}
\hline IF & $\tilde{a}_{0}$ & $\tilde{a}_{1}$ & $\tilde{a}_{2}$ & $\tilde{a}_{3}$ & Error/\% \\
\hline 1 & $1.0165 \mathrm{e}+06$ & $1.9574 \mathrm{e}+11$ & $2.9168 \mathrm{e}+14$ & $-5.9140 \mathrm{e}+18$ & 0.23 \\
2 & $9.9962 \mathrm{e}+06$ & $-1.4837 \mathrm{e}+11$ & $-1.1790 \mathrm{e}+14$ & $2.1988 \mathrm{e}+18$ & 1.09 \\
3 & $4.9995 \mathrm{e}+06$ & $2.0003 \mathrm{e}+11$ & $-1.9872 \mathrm{e}+15$ & $2.0296 \mathrm{e}+17$ & 3.86 \\
4 & $8.0149 \mathrm{e}+06$ & $8.2006 \mathrm{e}+10$ & $1.6507 \mathrm{e}+15$ & $-2.1428 \mathrm{e}+18$ & 8.78 \\
\hline
\end{tabular}

\section{Conclusions}

We present a novel separation method for the multi-radar signal based on PTFA. In the iterative process, five steps are addressed: initiation, identification with PTFA, demodulation, separation with adaptive filter and recovery. The proposed method has three advantages over the conventional methods. First, PTFA searches the optimal component in the entire time-frequency domain; this is effective for identifying weak radar signals. Second, an appropriate time-frequency kernel enables the method to characterize the signal with an accurate IF, which facilitates the signal separation. Finally, a bandwidth adaptive filter can cover as much as the energy of the interested component and reduce that of noise and other components. The experiment results show that the proposed method has good performance, which is promising in applications on electronic reconnaissance, e.g., electronic intelligence, electronic warfare support measures and radar warning.

\section{References}

[1] R. G. Wiley. ELINT: the interception and analysis of radar signals. Boston: Artech House, 2006.

[2] D. S. Jang, H. L. Choi, J. E. Roh. Optimization of surveillance beam parameters for phased array radars. Proc. of the IET International Conference on Radar Systems, 2012: 1-5.

[3] F. Gini, A. D. Maio, L. Patton. Waveform design and diversity for advanced radar systems. Herts, U.K.: Institution of Engneeying and Technology, 2011.

[4] K. Nishinuchi, M. Kobayashi. Improved algorithm for estimating pulse repetition intervals. IEEE Trans. on Aerospace and Electronic Systems, 2000, 56(3): 407-421.

[5] D. Zeng, X. Zeng, G. Lu, et al. Automatic modulation classification of radar signals using the generalised time-frequency representation of Zhao, Atlas and Marks. IET Radar, Sonar and Navigation, 2010, 5(4): 507-516.

[6] P. F. Pai. Online tracking of instantaneous frequency and amplitude of dynamical system response. Mechanical Systems and Signal Processing, 2010, 24(4): $1007-1024$.

[7] Y. Yang, Z. K. Peng, X. J. Dong, et al. Application of parameterized time-frequency analysis on multicomponent frequency modulated signals. IEEE Trans. on Instrumentation and Measurement, 2014, 63(12): 3169 - 3180 .

[8] X. Xia. A quantitative analysis of SNR in the short-time Fourier transform domain for multicomponent signals. IEEE Trans. on Signal Processing, 1998, 46(1): 200-203.

[9] J. Pons-Linares, J. A. Antonino-Daviu, et al. Induction motor diagnosis based on a transient current analytic wavelet transform via frequency b-splines. IEEE Trans. on Industrial Electronics, 2011, 58(5): 530-1544.

[10] V. C. Alarcon, J. A. Antonino-Daviu, M. Riera-Guasp, et al. Application of the Wigner-Ville distribution for the detection of rotor asymmetries and eccentricity through high-order harmonics. Electric Power Systems Research, 2012, 91: 28-36.

[11] H. K. Kwok, D. L. Jones. Improved instantaneous frequency estimation using an adaptive short-time fourier transform. IEEE Trans. on Signal Processing, 2000, 48(10): 2964-2972.

[12] B. P. Anderson. The rational resolution analysis: a generalization of multiresolution analyses with application to the specific emitter identification problem. USA: Air Force Institute of Technology, 1997.

[13] S. Stankovi, I. Djurovi. Motion parameter estimation by using time-frequency representations. Electronics Letter, 2001, 37: $1446-1448$.

[14] S. Barbarossa, A. Zanalda. A combined Wigner-Ville and Hough transform for cross-terms suppression and optimal detection and parameter estimation. IEEE Trans. on Acoustic, Speech and Signal Processing, 1992, 5: 173-176.

[15] S. Barbarossa. Analysis of multicomponent LFM signals by a combined Wigner-Hough transform. IEEE Trans. on Signal Processing, 1995, 43: $1511-1515$.

[16] L. Angrisani, M. D'Arco. A measurement method based on a modified version of the Chirplet transform for instantaneous frequency estimation. IEEE Trans. on Instrumentation and measurement, 2002, 51(4): 704-711.

[17] J. Cui, W. Wong. The adaptive Chirplet transform and visual evoked potentials. IEEE Trans. on Biomedical Engineering, 2006, 53(7): $1378-1384$.

[18] B. Dugnol, C. Fernandez, G. Galiano, et al. On a Chirplet transform-based method applied to separating and counting Wolfhowls. Signal Processing, 2012, 88: 1817-1826.

[19] L. Angrisani, M. D. Arco, R. S. Lo Moriello, et al. On the use of the Warblet transform for instantaneous frequency estimation. IEEE Trans. on Instrumentation and Measurement, 2005, 54(4): $1374-1380$. 
[20] Y. Yang, Z. K. Peng, X. J. Dong, et al. General parameterized time-frequency transform. IEEE Trans. on Signal Processing, 2014, 62: $2751-2764$.

[21] Y. Yang, W. M. Zhang, Z. K. Peng, et al. Multicomponent signal analysis based on polynomial Chirplet transform. IEEE Trans. on Industrial Electronics, 2013, 60(9): 3948 - 3956.

[22] Y. Yang, Z. K. Peng, G. Meng, et al. Spline-Kernelled Chirplet transform for the analysis of signals with time-varying frequency and its application. IEEE Trans. on Industrial Electronics, 2012, 59(3): 1612-1621.

[23] Y. Yang, Z. K. Peng, G. Meng, et al. Characterize highly oscillating frequency modulation using generalized Warblet transform. Mechanical Systems and Signal Processing, 2012, 26: $128-140$

[24] J. Terrien, C. Marque, G. Germain. Ridge extraction from the time-frequency representation (TFR) of signals based on an image processing approach: application to the analysis of uterine electromyogram AR TFR. IEEE Trans. on Biomedical Engineering, 2008, 55(5): 1496-1503.

[25] A. Francos, M. Porat. Analysis and synthesis of multicomponent signals using positive time-frequency distributions. IEEE Trans. on Signal Processing, 1999, 47(2): 493 - 514.

[26] Y. Yang, X. J. Dong, Z. K. Peng, et al. Component extraction for non-stationary multi-component signal using parameterized de-chirping and band-pass filter. IEEE Signal Processing Letters, 2015, 22(9): 1373 - 1377.

[27] H. Y. Lee, Z. Bien. A variable bandwidth filter for estimation of instantaneous frequency and reconstruction of signals with time-varying spectral content. IEEE Trans. on Signal Processing, 2011, 59(5): 2052-2071.

[28] H. Kar. A note on the improved LMI-based criterion for global asymptotic stability of 2-D state-space digital filters described by Roesser model using two's complement overflow arithmetic. Digital Signal Processing, 2013, 23: 1767 - 1772.

[29] S. Nakamori. Design of RLS-FIR filter using covariance information in linear continuous-time stochastic systems. Applied Mathematics and Computation, 2013, 219: 9598-9608.

[30] S. B. Wang, X. F. Chen, Y. Wang, et al. Nolinear squeezing time-frequency transform for weak signal detection. Signal Processing, 2015, 113: 195-210.

\section{Biographies}
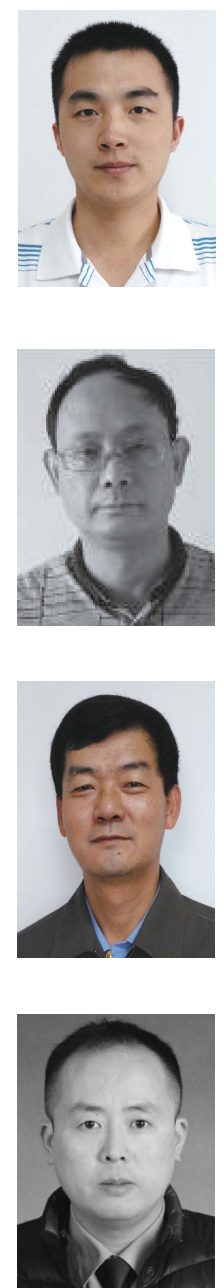

Wenlong Lu was born in 1988. He received his M.S. degree from Air Force Engineering University in 2012. Currently he is studying in Air Force Engineering University as a Ph.D. candidate. His research interests include electronic war (EW) techniques based on stealthy platform and microelectronic techniques applying to EW systems.

E-mail: youranqixia521@126.com

Junwei Xie was born in 1970. He received his Ph.D. degree from Air Force Engineering University. Currently he is working in Air Force Engineering University as a professor. His research interests include electronic jamming and anti-jamming and radar environment simulation.

E-mail: jun_wei_xie@126.com

Heming Wang was born in 1965. He received his M.S. degree from Air Force Engineering University. Currently he is working in Air Force Engineering University as a professor. His research interests include microelectronic techniques applying to defense systems.

E-mail: heming_wang@126.com

Chuan Sheng was born in 1979. He received his M.S. degree from Air Force Engineering University. Currently he is working in Air Force Engineering University as an associate professor. His research interests include electronic jamming and antijamming and radar environment simulation.

E-mail: hidaicy@126.com 